\title{
The Cramer-Rao Bound for Channel Estimation in Block Fading Amplify-and-Forward Relaying Networks
}

\author{
Nico Aerts, Iancu Avram and Marc Moeneclaey \\ Department of Telecommunications \\ and Information Processing \\ Ghent University \\ 9000 Gent, Belgium \\ Email: firstname.lastname@telin.ugent.be
}

\begin{abstract}
In this paper, we express the Cramer-Rao Bound (CRB) for channel coefficient and noise variance estimation at the destination of an Amplify-and- Forward (AF) based cooperative system, in terms of the a posteriori expectation of the codewords. An algorithm based on factor graphs can be applied in order to calculate this expectation. As the computation of the CRB is rather intensive, the modified CRB (MCRB), which is a looser bound, is derived in closed form. It can be shown that the MCRB coincides with the CRB in the high signal-to-noise ratio (SNR) limit and to that end the CRB/MCRB ratio is simulated in case of uncoded and convolutional encoded transmission.

Index Terms-cooperative communication, Amplify-andForward, Cramer-Rao Bound, modified CRB
\end{abstract}

\section{INTRODUCTION}

Cooperative communication [1], [2] is a promising and relatively new method to obtain spatial diversity in a wireless network, involving other terminals, which we refer to as relays, that transmit to the destination information that is related to the message sent by the source. Often, the cooperative protocols proposed in literature are investigated under the assumption that all channel state information is available at the destination [3-6]. However, in a realistic scenario, channel estimates need to be derived at the destination. The goal of this paper is to establish lowerbounds on the performance of practical channel parameter estimators, in case of the Amplify-and-Forward (AF) protocol. These bounds can serve as benchmarks for practical channel estimation algorithms.

If unbiased estimates of the channel parameters are obtainable, a well known lower bound on the mean-square estimation error is the Cramer-Rao Bound (CRB) [7]. The received signal contains not only the channel parameters to be estimated, but also unknown coded symbols that convey the digital information. As far as the channel estimation is concerned, these symbols are considered as nuisance parameters. We show that the CRB can be expressed as a function of the a posteriori expectation of the coded symbols. Message passing on a factor graph [8] can be used to obtain the marginal a posteriori probabilities of the coded symbols, from which their a posteriori expectation is easily derived. In the case of a convolutional code, this approach reduces to the BCJR algorithm [9], which requires a forward and a backward recursion on the trellis to obtain the a posteriori probabilities. Further, we derive a closed-form expression for the modified CRB (MCRB) [10], which accurately captures the high-SNR behavior of the CRB.

\section{System Description}

In a cooperative system with $M$ relays, the time frame allocated to the source is divided into $M+1$ time slots. Only the first slot is utilized by the source to broadcast information; the remaining $M$ slots are used by the relays. As the name of the amplify-and-forward protocol implies, the $\mathrm{i}^{\text {th }}$ relay amplifies the signal received from the source, and forwards it to the destination during time slot $i+1(i=1, \ldots, M)$. In this contribution we restrict ourselves to systems with only one relay. The network then consists of a source $S$, a relay $R$ and a destination D as depicted in Fig. 1. Extension of our results to the case of multiple relays is straightforward, as the source and the relays all transmit in nonoverlapping intervals.

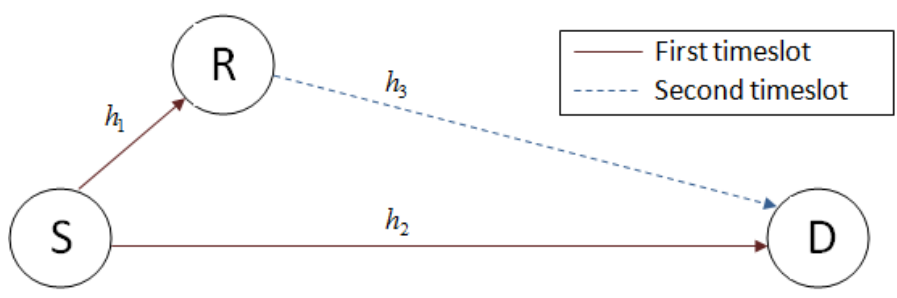

Fig. 1. Network containing a source $\mathrm{S}$, a relay $\mathrm{R}$ and a destination D.

At the source, an error correcting code [11] is used to encode the vector of information bits $\boldsymbol{b}$. The result after mapping onto the symbol constellation is the codeword $c$, which is a row vector of $K_{d}$ coded symbols. We assume slow independent frequency flat Rayleigh fading channels with additive white Gaussian noise, so the signals received by the 
relay and the destination during the first time slot are given by

$$
\begin{aligned}
& \boldsymbol{r}_{\boldsymbol{S}}=h_{1} \boldsymbol{c}+\boldsymbol{n}_{\mathbf{1}}, \\
& \boldsymbol{d}_{\boldsymbol{S}}=h_{2} \boldsymbol{c}+\boldsymbol{n}_{\mathbf{2}},
\end{aligned}
$$

respectively. Here the channel coefficients $h_{m}$ and the elements of the noise vectors $\boldsymbol{n}_{\boldsymbol{m}}$ are independent ZMCSCG (zero-mean circular symmetric complex Gaussian) distributed with variances $H_{m}$ and $N_{m}$, respectively, with $m \in\{1,2\}$. The gain factor $\beta$ at the relay is chosen as

$$
\beta=\sqrt{\frac{E_{r}}{H_{1} E_{s}+N_{1}}},
$$

in order to fulfill the following power constraint :

$$
\frac{\mathbb{E}\left[\left|\beta \boldsymbol{r}_{\boldsymbol{S}}\right|^{2}\right]}{K_{d}}=E_{r},
$$

where $\mathbb{E}[x]$ denotes the statistical expectation of $x$, while $E_{s}$ and $E_{r}$ denote the average energy per symbol transmitted by the source and the relay, respectively. The signal received by the destination during the second time slot can then be written as

$$
\begin{aligned}
\boldsymbol{d}_{\boldsymbol{R}} & =h_{3} \beta \boldsymbol{r}_{\boldsymbol{S}}+\boldsymbol{n}_{\mathbf{3}} \\
& =\beta h_{4} \boldsymbol{c}+\boldsymbol{n}_{\mathbf{4}},
\end{aligned}
$$

where $h_{3}$ and the elements of $\boldsymbol{n}_{\mathbf{3}}$ are ZMCSCG distributed with variances $H_{3}$ and $N_{3}$, the channel gain on the S-R-D link is $h_{4}=h_{1} h_{3}$, and the elements of the noise $\boldsymbol{n}_{\mathbf{4}}=\beta h_{3} \boldsymbol{n}_{\mathbf{1}}+\boldsymbol{n}_{\mathbf{3}}$ on the S-R-D link are ZMCSCG distributed with variances $N_{4}=\left|h_{3}\right|^{2} \beta^{2} N_{1}+N_{3}$; note that $N_{4}$ depends on the realization of the channel gain $h_{3}$ on the R-D link. We will assume the noise variances $N_{m}$ to be known $(m=1,2,3)$ so the destination has to estimate the channel parameters $h_{2}, h_{4}$ and $N_{4}$ in order to be able to decode the information from the source.

\section{LOWERBOUNDS ON MSEE}

In this section we will derive the CRB and the MCRB for joint estimation of the parameters $h_{2}, h_{4}$ and $N_{4}$, which we will group into the real valued vector $\boldsymbol{x}=$ $\left(\Re\left\{h_{2}\right\}, \Im\left\{h_{2}\right\}, \Re\left\{h_{4}\right\}, \Im\left\{h_{4}\right\}, N_{4}\right)$. We assume that the destination considers $\boldsymbol{x}$ as an unknown deterministic parameter (i.e., the destination ignores the a priori distribution of $\boldsymbol{x}$ ). Furthermore we denote $\boldsymbol{d}=\left(\boldsymbol{d}_{\boldsymbol{S}}, \boldsymbol{d}_{\boldsymbol{R}}\right)$ and assume $\hat{\boldsymbol{x}}$ to be an unbiased estimate of $\boldsymbol{x}$. For a given realization of $\boldsymbol{x}$, the meansquare estimation error (MSEE) related to the $i$ th element $\hat{x}_{i}$ $(i=1 \ldots 5)$ is now bounded by

$$
\mathbb{E}_{\boldsymbol{d}}\left[\left(x_{i}-\hat{x}_{i}\right)^{2}\right] \geq C R B_{i}(\boldsymbol{x}) \geq M C R B_{i}(\boldsymbol{x}),
$$

where $C R B_{i}$ and $M C R B_{i}$ are defined below, and $\mathbb{E}_{\boldsymbol{d}}$ is the statistical expectation with respect to the pdf $p(\boldsymbol{d} ; \boldsymbol{x})$ of $\boldsymbol{d}$, which contains $\boldsymbol{x}$ as a parameter. Note that (5) depends on the particular realization of $\boldsymbol{x}$. This dependency can be removed by averaging (5) over $\boldsymbol{x}$, yielding

$$
\mathbb{E}\left[\left(x_{i}-\hat{x}_{i}\right)^{2}\right] \geq \mathbb{E}_{\boldsymbol{x}}\left[C R B_{i}(\boldsymbol{x})\right] \geq \mathbb{E}_{\boldsymbol{x}}\left[M C R B_{i}(\boldsymbol{x})\right] .
$$

According to the definition of $\boldsymbol{x}$, we have

$$
\begin{aligned}
\left|h_{2}-\hat{h}_{2}\right|^{2} & =\left(x_{1}-\hat{x}_{1}\right)^{2}+\left(x_{2}-\hat{x}_{2}\right)^{2}, \\
\left|h_{4}-\hat{h}_{4}\right|^{2} & =\left(x_{3}-\hat{x}_{3}\right)^{2}+\left(x_{4}-\hat{x}_{4}\right)^{2}, \\
\left(N_{4}-\hat{N}_{4}\right)^{2} & =\left(x_{5}-\hat{x}_{5}\right)^{2},
\end{aligned}
$$

such that lower bounds on the MSEEs for $h_{2}, h_{4}$ and $N_{4}$ follow easily from (5) and (6).

\section{A. Cramer-Rao Bound}

The $(i, j)$ th element of the Fisher information matrix [7] $\mathrm{J}(\boldsymbol{x})$ related to the estimation of $\boldsymbol{x}$ is given by

$$
\mathrm{J}_{i, j}(\boldsymbol{x})=\mathbb{E}_{\boldsymbol{d}}\left[\frac{\partial \ln p(\boldsymbol{d} ; \boldsymbol{x})}{\partial x_{i}} \cdot \frac{\partial \ln p(\boldsymbol{d} ; \boldsymbol{x})}{\partial x_{j}}\right] .
$$

The corresponding $C R B_{i}(\boldsymbol{x})$ is obtained as

$$
C R B_{i}(\boldsymbol{x})=\left(\mathrm{J}^{-1}(\boldsymbol{x})\right)_{i, i} .
$$

Taking into account that

$$
p(\boldsymbol{d} ; \boldsymbol{x})=\frac{1}{|\mathrm{C}|} \sum_{\tilde{\boldsymbol{c}} \in \mathrm{C}} p(\boldsymbol{d} \mid \boldsymbol{c}=\tilde{\boldsymbol{c}} ; \boldsymbol{x}),
$$

where $C$ denotes the set of codewords with cardinality $|C|$, one obtains

$$
\frac{\partial \ln p(\boldsymbol{d} ; \boldsymbol{x})}{\partial x_{i}}=\sum_{\tilde{\boldsymbol{c}} \in \mathrm{C}} \operatorname{Pr}[\boldsymbol{c}=\tilde{\boldsymbol{c}} \mid \boldsymbol{d} ; \boldsymbol{x}] \frac{\partial \ln p(\boldsymbol{d} \mid \boldsymbol{c}=\tilde{\boldsymbol{c}} ; \boldsymbol{x})}{\partial x_{i}},
$$

where

$$
\operatorname{Pr}[\boldsymbol{c}=\tilde{\boldsymbol{c}} \mid \boldsymbol{d} ; \boldsymbol{x}]=\frac{p(\boldsymbol{d} \mid \boldsymbol{c}=\tilde{\boldsymbol{c}} ; \boldsymbol{x})}{\sum_{\overline{\boldsymbol{c}} \in \mathrm{C}} p(\boldsymbol{d} \mid \boldsymbol{c}=\overline{\boldsymbol{c}} ; \boldsymbol{x})}
$$

denotes the a posteriori distribution of the codewords. For given $c$ and $x$, it follows from (1) and (4) that $\boldsymbol{d}_{S}$ and $\boldsymbol{d}_{\boldsymbol{R}}$ are statistically independent Gaussian vectors with means $h_{2} c$ and $\beta h_{4} c$, respectively; their components are statistically independent with variances $N_{2}$ and $N_{4}$, respectively. From this, we obtain ${ }^{1}$

$$
\begin{aligned}
& \frac{\partial \ln p(\boldsymbol{d} ; \boldsymbol{x})}{\partial x_{1}}+j \frac{\partial \ln p(\boldsymbol{d} ; \boldsymbol{x})}{\partial x_{2}}=\frac{2}{N_{2}}\left(\boldsymbol{d}_{\boldsymbol{S}} \boldsymbol{u}^{H}-h_{2} g\right) \\
& \frac{\partial \ln p(\boldsymbol{d} ; \boldsymbol{x})}{\partial x_{3}}+j \frac{\partial \ln p(\boldsymbol{d} ; \boldsymbol{x})}{\partial x_{4}}=\frac{2 \beta}{N_{4}}\left(\boldsymbol{d}_{\boldsymbol{R}} \boldsymbol{u}^{H}-\beta h_{4} g\right) \\
& \frac{\partial \ln p(\boldsymbol{d} ; \boldsymbol{x})}{\partial x_{5}}=\frac{1}{N_{4}^{2}}\left(\left|\boldsymbol{d}_{\boldsymbol{R}}-\beta h_{4} \boldsymbol{u}\right|^{2}+\beta^{2}\left|h_{4}\right|^{2}\left(g-|\boldsymbol{u}|^{2}\right)-K_{d} N_{4}\right)
\end{aligned}
$$

where $\boldsymbol{u}$ and $g$ denote the a posteriori expectation of $\boldsymbol{c}$ and $|c|^{2}$, respectively:

$$
\boldsymbol{u}=\frac{\sum_{\overline{\boldsymbol{c}} \in \mathrm{C}} \overline{\boldsymbol{c}} p(\boldsymbol{d} \mid \overline{\boldsymbol{c}} ; \boldsymbol{x})}{\sum_{\tilde{\boldsymbol{c}} \in \mathrm{C}} p(\boldsymbol{d} \mid \tilde{\boldsymbol{c}} ; \boldsymbol{x})}
$$

and

$$
g=\frac{\sum_{\overline{\boldsymbol{c}} \in \mathrm{C}}|\overline{\boldsymbol{c}}|^{2} p(\boldsymbol{d} \mid \overline{\boldsymbol{c}} ; \boldsymbol{x})}{\sum_{\tilde{\boldsymbol{c}} \in \mathrm{C}} p(\boldsymbol{d} \mid \tilde{\boldsymbol{c}} ; \boldsymbol{x})} .
$$

\footnotetext{
${ }^{1}$ more details in appendix A
} 
As stated before, both $\boldsymbol{u}$ and $g$ are easily derived from the marginal a posteriori probabilities of the coded symbols, which are obtainable by message passing on a factor graph. Indeed

$$
\begin{aligned}
u(l) & =\sum_{s \in \mathbb{S}} s p(c(l)=s \mid d(l) ; \boldsymbol{x}), \\
g & =\sum_{l=1}^{K_{d}} \sum_{s \in \mathbb{S}}|s|^{2} p(c(l)=s \mid d(l) ; \boldsymbol{x}),
\end{aligned}
$$

with $\mathbb{S}$ the set of constellation points and $u(l), c(l)$ and $d(l)$ denoting the $l$ th element of $\boldsymbol{u}, \boldsymbol{c}$ and $\boldsymbol{d}$, respectively. Note that if all codewords have the same energy ${ }^{2}, g$ equals $K_{d} E_{s}$. In order to obtain $C R B_{i}(\boldsymbol{x})$ for $i=1, \ldots, 5$, the following steps are carried out:

(i) Generate $\mathrm{N}$ independent realizations of $\left(\boldsymbol{c}, \boldsymbol{n}_{\mathbf{1}}, \boldsymbol{n}_{\mathbf{2}}, \boldsymbol{n}_{\mathbf{3}}\right)$, and compute the corresponding realizations of $\boldsymbol{d}=\left(\boldsymbol{d}_{\boldsymbol{S}}, \boldsymbol{d}_{\boldsymbol{R}}\right)$ for given $\boldsymbol{x}$ according to (1) and (4).

(ii) For each realization of $\boldsymbol{d}$, compute the corresponding expression between brackets from (10), making use of (15).

(iii) Compute the elements of $\mathrm{J}(\boldsymbol{x})$ by replacing the expectation from (10) by an arithmetical average over the $\mathrm{N}$ realizations of $\boldsymbol{d}$. For sufficiently large $\mathrm{N}$, the arithmetical average converges to the expectation.

(iv) Compute $C R B_{i}(\boldsymbol{x})$ according to (11).

In order to obtain the average $\mathbb{E}_{\boldsymbol{x}}\left[C R B_{i}(\boldsymbol{x})\right]$, the above procedure must be executed for a number of different realizations of $\boldsymbol{x}$, and the arithmetical average of the corresponding $C R B_{i}(\boldsymbol{x})$ must be computed.

\section{B. Modified Cramer-Rao Bound}

The evaluation of the true CRB is computationally intensive, due to the presence of the unknown codewords. The MCRB, which is looser than the CRB, is obtained in a similar way as the CRB, but with the Fisher information matrix $\mathrm{J}(\boldsymbol{x})$ replaced by the modified Fisher information matrix $\mathrm{J}_{M}(\boldsymbol{x})$. The elements of $\mathrm{J}_{M}(\boldsymbol{x})$ are given by [10]:

$$
\left(\mathrm{J}_{M}(\boldsymbol{x})\right)_{i, j}=\mathbb{E}_{\boldsymbol{c}}\left[\mathbb{E}_{\boldsymbol{d} \mid \boldsymbol{c}}\left[\frac{\partial \ln p(\boldsymbol{d} \mid \boldsymbol{c} ; \boldsymbol{x})}{\partial x_{i}} \cdot \frac{\partial \ln p(\boldsymbol{d} \mid \boldsymbol{c} ; \boldsymbol{x})}{\partial x_{j}}\right]\right] .
$$

where $\mathbb{E}_{\boldsymbol{d} \mid \boldsymbol{c}}[$.$] and \mathbb{E}_{\boldsymbol{c}}[$.$] denote expectation w.r.t. p(\boldsymbol{d} \mid \boldsymbol{c} ; \boldsymbol{x})$ and averaging over the codewords, respectively. As $p(\boldsymbol{d} \mid \boldsymbol{c} ; \boldsymbol{x})$ is Gaussian, $\mathrm{J}_{M}(\boldsymbol{x})$ and $M C R B_{i}(\boldsymbol{x})$ can be obtained in closed form. It turns out that $\mathrm{J}_{M}(\boldsymbol{x})$ is diagonal ${ }^{3}$, yielding

$$
\begin{aligned}
\mathbb{E}_{\boldsymbol{d}}\left[\left|h_{2}-\hat{h}_{2}\right|^{2}\right] & \geq \frac{N_{2}}{K_{d} E_{s}}, \\
\mathbb{E}_{\boldsymbol{d}}\left[\left|h_{4}-\hat{h}_{4}\right|^{2}\right] & \geq \frac{N_{4}}{\beta^{2} K_{d} E_{s}}, \\
\mathbb{E}_{\boldsymbol{d}}\left[\left|N_{4}-\hat{N}_{4}\right|^{2}\right] & \geq \frac{N_{4}^{2}}{K_{d}} .
\end{aligned}
$$

Note that the MCRB related to $h_{2}$ does not depend on $h_{2}$, whereas the MCRBs related to $h_{4}$ and $N_{4}$ both depend on $N_{4}$, but not on $h_{4}$. Averaging both sides of (18) w.r.t. $N_{4}$ yields the average MCRBs; these are obtained by simply

\footnotetext{
${ }^{2}$ e.g. in case M-PSK mapping is used

${ }^{3}$ more details in appendix B
}

replacing in (18) the quantities $N_{4}$ (2nd equation) and $N_{4}^{2}$ (3rd equation) by $\mathbb{E}\left[N_{4}\right]=H_{3} \beta^{2} N_{1}+N_{3}$ and $\mathbb{E}\left[N_{4}^{2}\right]=$ $\left(H_{3} \beta^{2} N_{1}+N_{3}\right)^{2}+H_{3}^{2} \beta^{4} N_{1}^{2}$, respectively. Note that these results are code independent.

\section{NUMERICAL RESUlTS}

In [12] it has been shown that the MCRB coincides with the CRB at the high signal-to-noise (SNR) limit. We compute the $\mathrm{CRB} / \mathrm{MCRB}$ ratio in case of the terminated rate- $\frac{1}{2}(15,17)$ convolutional code (a) and the uncoded situation (b). BPSK mapping is used and we choose $K_{d}=260$ and $H_{1}=H_{2}=$ $H_{3}=1$. Furthermore we assume $N_{1}=N_{3}=2 N_{2}$ and the power constraints at the source and the relay to be identical, i.e. $E_{r}=E_{s}$. In the uncoded case the a posteriori expectation is derived analytically as

$$
\boldsymbol{u}=\sqrt{E_{s}} \tanh \left(2 \sqrt{E_{s}} \Re\left\{\frac{\beta h_{4}^{*} \boldsymbol{d}_{\boldsymbol{R}}}{N_{4}}+\frac{h_{2}^{*} \boldsymbol{d}_{\boldsymbol{S}}}{N_{2}}\right\}\right),
$$

whereas the BCJR algorithm is used in the case of convolutional coding. The results for each of the parameters $h_{2}, h_{4}$ and $N_{4}$ are now depicted in Fig. 2. Here we have averaged over 5000 different parameter sets $\left(h_{1}, h_{2}, h_{3}\right)$ and for each parameter set 1000 frames are sent. We observe that the degradation of the CRB as compared to the MCRB increases with decreasing SNR as the a posteriori expectation deviates more from the actual symbols. This degradation is larger in the uncoded situation and when estimating the channel coefficients than when estimating the noise variance. We now consider the situation where a rate- $\frac{1}{3}(15,17,13)$

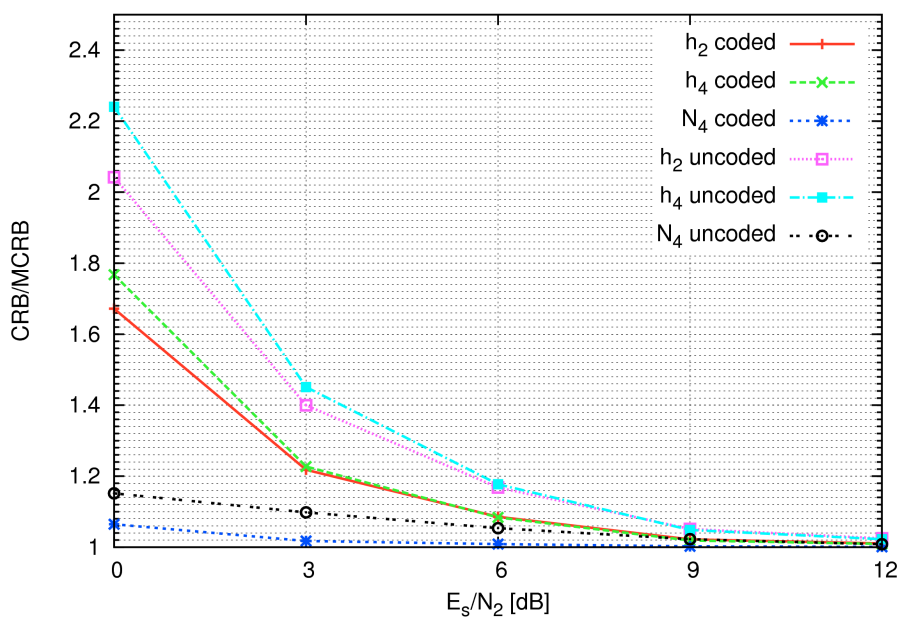

Fig. 2. The ratio CRB/MCRB for the parameters $h_{2}, h_{4}$ and $N_{4}$ in case of BPSK mapping.

convolutional code and 8-PSK mapping is used. As the MCRB is also independent of the considered constellation, we expect the corresponding CRBs to have the same high SNR limit as in case (a), where BPSK mapping is used. Fig. 3 depicts these bounds for parameters $h_{4}$ and $N_{4}$ and we note again that estimating the noise variance is less sensitive to the deviation of the a posteriori expectation from the actual 


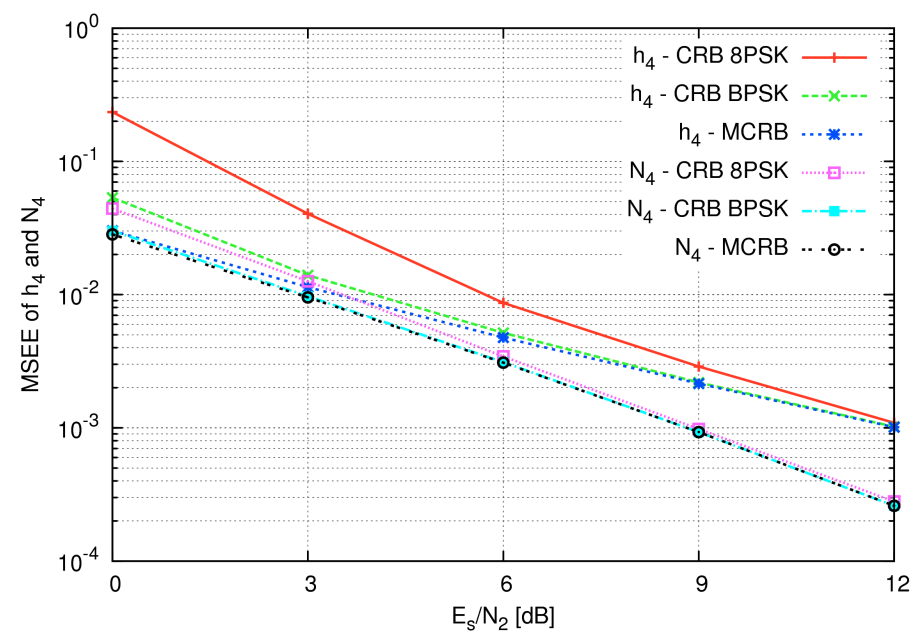

Fig. 3. The CRB and MCRB related to the estimation of $h_{2}, h_{4}$ and $N_{4}$.

symbols.

In case of 4-PSK mapping, Fig. 4 and Fig. 5 depict the MCRB and the CRB related to the estimation of $h_{4}$ and $N_{4}$, respectively, in the coded and uncoded situation. Now observe the small difference between the uncoded and coded situation, suggesting that it is possible to find an unbiased estimator which ignores the encoder constrains and has a similar performance as an unbiased estimator which exploits the properties of the code. Considering the expression of the a posteriori expectation of the codewords, the performance in the uncoded case is related to the minimum Euclidean distance between the symbols wheras the minimum Euclidean distance between codewords is the determining factor in the coded case. As a result, a similar performace can only be achieved if a small constellation is used.

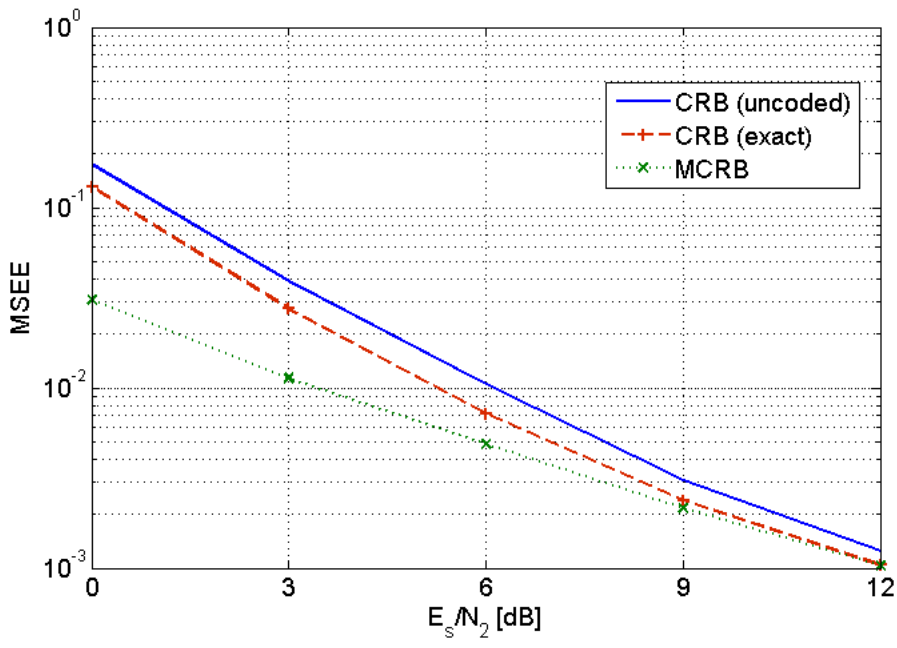

Fig. 4. The CRB related to the estimation of $h_{4}$ in the coded and uncoded situation and in case of 4-PSK mapping.

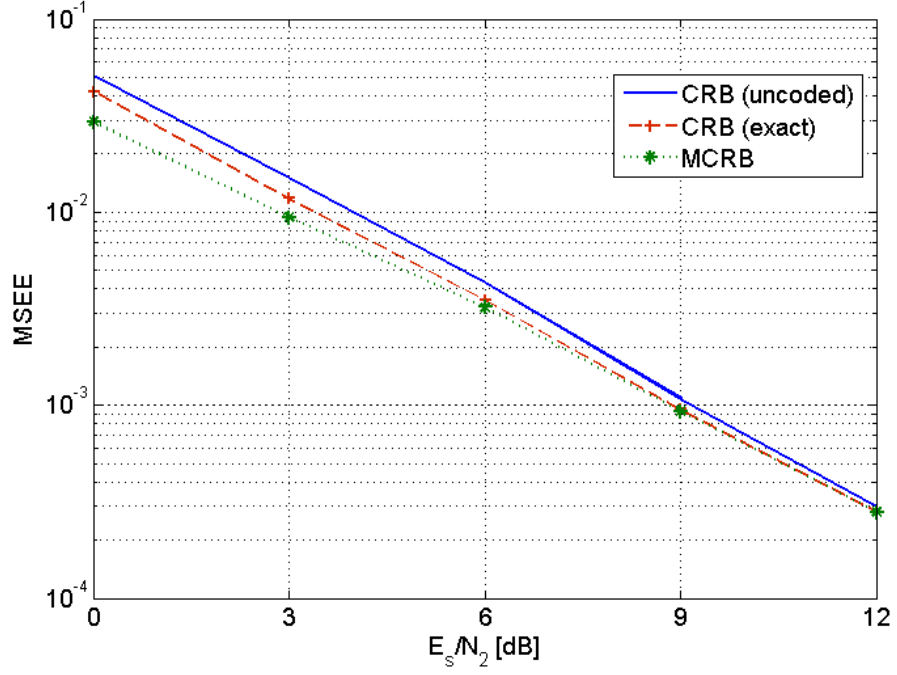

Fig. 5. The CRB related to the estimation of $N_{4}$ in the coded and uncoded situation and in case of 4-PSK mapping.

In order to translate these bounds on the MSEE into bounds on the Frame Error Rate (FER), we consider a genie estimator for each of the parameters $h_{2}, h_{4}$ and $N_{4}$. The genie estimator related to a parameter (e.g., $N_{4}$ ) performs maximum-likelihood estimation under the assumption that the other parameters (i.e., $h_{2}$ and $h_{4}$ ) and the data symbols $c$ are known. It is now easily seen that the corresponding estimates listed below are unbiased and their MSEEs coincide with the MCRBs.

$$
\begin{aligned}
& \hat{h}_{2}=\frac{\boldsymbol{d}_{S} \boldsymbol{c}^{H}}{|\boldsymbol{c}|^{2}}, \\
& \hat{h}_{4}=\frac{\boldsymbol{d}_{\boldsymbol{R}} \boldsymbol{c}^{H}}{\beta|\boldsymbol{c}|^{2}}, \\
& \hat{N}_{4}=\frac{\left|\boldsymbol{d}_{\boldsymbol{R}}-h_{4} \beta \boldsymbol{c}\right|^{2}}{K_{d}} .
\end{aligned}
$$

For 4-PSK, Fig. 6 depicts the genie lowerbound on the FER, which results from a decoder that uses the above genie estimates. Also shown is the FER curve corresponding to perfect channel knowledge at the destination. As the MCRB captures the high SNR behavior of the CRB, this result indicates that an estimator with a MSEE approaching the CRB yields a highSNR FER that is close to the FER corresponding to perfect channel knowledge.

\section{Conclusions}

In this contribitions we have expressed the Cramer-Rao bound for joint channel coefficient and noise variance estimation, in an Amplify-and-Forward (AF) based cooperative system, in terms of the a posteriori expectation of the code words. These expectations can be derived by message passing on a factor graph, which reduces to the BCJR algorithm in case of convolutional codes. As the computations to obtain this true CRB are rather intensive, the modified CRB is derived analytically. Both bounds can now be used as benchmarks 


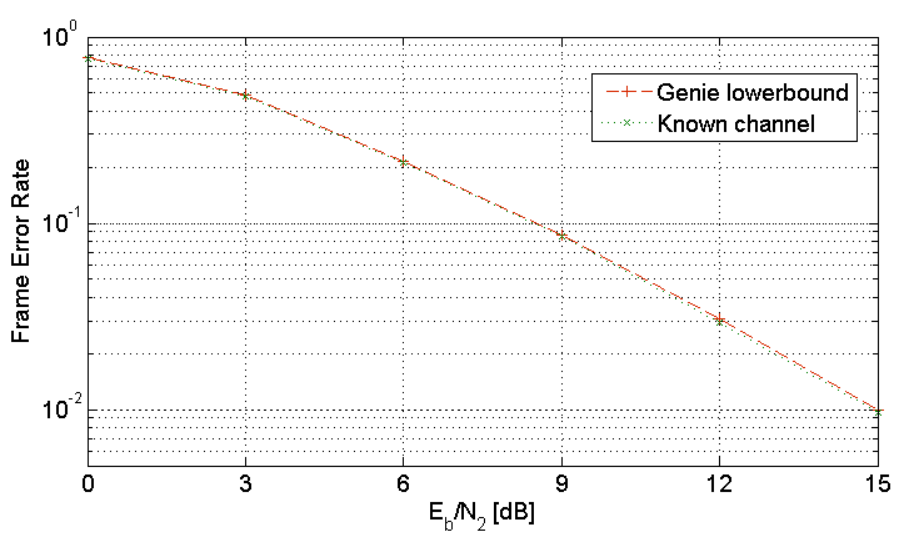

Fig. 6. FER in case of a $(15,17)$ convolutional code and 4-PSK mapping.

for the MSEE resulting from practical channel estimation algorithms.

\section{APPENDIX A}

In order to obtain (15), it follows from (13) that we need expressions for $\frac{\partial \ln p(\boldsymbol{d} \mid \boldsymbol{c} ; \boldsymbol{x})}{\partial x_{i}},(i=1 \ldots .5)$. Due to similarities we will only present the partial derivatives w.r.t. $x_{3}$ and $x_{5}$. Given (1) and (4) we obtain

$$
p(\boldsymbol{d} \mid \boldsymbol{c} ; \boldsymbol{x})=\frac{1}{\left(\pi^{2} N_{2} N_{4}\right)^{K_{d}}} e^{\frac{-\left|d_{R}-\beta h_{4} c\right|^{2}}{N_{4}}+\frac{-\left|d_{S}-h_{2} c\right|^{2}}{N_{2}}}
$$

so

$$
\frac{\partial \ln p(\boldsymbol{d} \mid \boldsymbol{c} ; \boldsymbol{x})}{\partial x_{3}}=\frac{-2 \beta^{2} x_{3}|\boldsymbol{c}|^{2}+\beta \boldsymbol{c} \boldsymbol{d}_{\boldsymbol{R}}^{H}+\beta \boldsymbol{d}_{\boldsymbol{R}} \boldsymbol{c}^{H}}{N_{4}}
$$

and

$$
\begin{aligned}
\frac{\partial \ln p(\boldsymbol{d} \mid \boldsymbol{c} ; \boldsymbol{x})}{\partial x_{5}} & =\frac{-K_{d}}{N_{4}}+\frac{\left|\boldsymbol{d}_{\boldsymbol{R}}-\beta h_{4} \boldsymbol{c}\right|^{2}}{N_{4}^{2}} \\
& =\frac{-K_{d}}{N_{4}}+\frac{\left|\boldsymbol{d}_{\boldsymbol{R}}\right|^{2}+\beta^{2} h_{4}^{2}|\boldsymbol{c}|^{2}-2 \beta \Re\left\{h_{4} \boldsymbol{c} \boldsymbol{d}_{\boldsymbol{R}}^{H}\right\}}{N_{4}^{2}} .
\end{aligned}
$$

Now making use of (16) and (17), we find

$$
\frac{\partial \ln p(\boldsymbol{d} ; \boldsymbol{x})}{\partial x_{3}}=\frac{2 \beta \Re\left\{\boldsymbol{d}_{\boldsymbol{R}} \boldsymbol{u}^{H}-\beta h_{4} g\right\}}{N_{4}}
$$

and

$$
\frac{\partial \ln p(\boldsymbol{d} ; \boldsymbol{x})}{\partial x_{5}}=\frac{-K_{d}}{N_{4}}+\frac{\left|\boldsymbol{d}_{\boldsymbol{R}}\right|^{2}+\beta^{2} h_{4}^{2} g-2 \beta \Re\left\{h_{4} \boldsymbol{u} \boldsymbol{d}_{\boldsymbol{R}}^{H}\right\}}{N_{4}^{2}} .
$$

\section{APPENDIX B}

The modified Fisher information matrix is given by

$$
\mathrm{J}_{M}(\boldsymbol{x})=\mathbb{E}_{\boldsymbol{d}}\left[\left(\begin{array}{c}
\frac{2 \Re\left\{\boldsymbol{n}_{\mathbf{2}} \boldsymbol{c}^{H}\right\}}{N_{2}} \\
\frac{2 \Im\left\{\boldsymbol{n}_{\mathbf{2}} \boldsymbol{c}^{H}\right\}}{N_{2}} \\
\frac{2 \beta \Re\left\{\boldsymbol{n}_{\mathbf{4}} \boldsymbol{c}^{H}\right\}}{N_{4}} \\
\frac{2 \beta \Im\left\{\boldsymbol{n}_{\mathbf{4}} \boldsymbol{c}^{H}\right\}}{N_{4}} \\
-\frac{K_{d}}{N_{4}}+\frac{\left|\boldsymbol{n}_{4}\right|^{2}}{N_{4}^{2}}
\end{array}\right)\left(\begin{array}{c}
\frac{2 \Re\left\{\boldsymbol{n}_{\mathbf{2}} \boldsymbol{c}^{H}\right\}}{N_{2}} \\
\frac{2 \Im\left\{\boldsymbol{n}_{\mathbf{2}} \boldsymbol{c}^{H}\right\}}{N_{2}} \\
\frac{2 \beta \Re\left\{\boldsymbol{n}_{\mathbf{4}} \boldsymbol{c}^{H}\right\}}{N_{4}} \\
\frac{2 \beta \Im\left\{\boldsymbol{n}_{\mathbf{4}} \boldsymbol{c}^{H}\right\}}{N_{4}} \\
-\frac{K_{d}}{N_{4}}+\frac{\left|\boldsymbol{n}_{\mathbf{4}}\right|^{2}}{N_{4}^{2}}
\end{array}\right)^{T}\right]
$$

and the results from section III-B are easily obtainable. We will restrict ourself to the calculation of $\left(\mathrm{J}_{M}(\boldsymbol{x})\right)_{3,5}$ :

$$
\begin{aligned}
\left(\mathrm{J}_{M}(\boldsymbol{x})\right)_{3,5} & =\mathbb{E}_{\boldsymbol{d}}\left[\left(\frac{2 \beta \Re\left\{\boldsymbol{n}_{\mathbf{4}} \boldsymbol{c}^{H}\right\}}{N_{4}}\right)\left(-\frac{K_{d}}{N_{4}}+\frac{\left|\boldsymbol{n}_{\mathbf{4}}\right|^{2}}{N_{4}^{2}}\right)\right] \\
& =\frac{2 \beta}{N_{4}^{3}} \mathbb{E}_{\boldsymbol{d}}\left[\left(\Re\left\{\boldsymbol{n}_{\mathbf{4}} \boldsymbol{c}^{H}\right\}\right)\left(-K_{d} N_{4}+\left|\boldsymbol{n}_{\mathbf{4}}\right|^{2}\right)\right] \\
& =\frac{2 \beta}{N_{4}^{3}} \mathbb{E}_{\boldsymbol{d}}\left[\left(\Re\left\{\boldsymbol{n}_{\mathbf{4}} \boldsymbol{c}^{H}\right\}\right)\left|\boldsymbol{n}_{\mathbf{4}}\right|^{2}\right] \\
& =\frac{2 \beta}{N_{4}^{3}} E_{d}\left[\sum_{i=1}^{K_{d}} \Re\left\{n_{4}(i)\right\}^{3} \Re\{c(i)\}+\right. \\
\left.\sum_{i=1}^{K_{d}} \Im\left\{n_{4}(i)\right\}^{3} \Im\{c(i)\}\right] & \\
& =0
\end{aligned}
$$

Here we used the notation $y(i)$ to denote the $\mathrm{i}^{\text {th }}$ element of the vector $\boldsymbol{y}$.

\section{REFERENCES}

[1] J. N. Laneman, "Cooperative Diversity in Wireless networks: Algorithms and Architectures," Ph.D. dissertation, Massachusetts Institute of Technology, Massachusetts, USA, 2002

[2] A. Sendonaris, E. Erkip and B. Aazhang, "User Cooperation DiversityPart I: System Description," IEEE Trans. Commun., vol. 51, no. 11, pp. 1927-1938, Nov. 2003

[3] T. Cover and A. E. Gamal, "Capacity Theorems for the Relay Channel," IEEE Trans. Inform. Theory, vol. 25, no. 5, pp. 572-584, Sep. 1979

[4] M. Souryal and H. You, "Quantize-and-Forward Relaying with Mary Phase Shift Keying," Wireless Communication and Networking Conference, 2008, pp. 42-47

[5] B. Djeumou, S. Lasaulce, and A. G. Klein "Practical quantize-andforward schemes for the frequency division relay channel," EURASIP Journal on Wireless Communications and Networking, vol. 2007, no. 2, Oct. 2007

[6] T. Hunter and A. Nosratinia, "Diversity through Coded Cooperation," IEEE Trans. Wireless Commun., vol. 5, no. 2, pp. 283-289, Feb. 2006

[7] H. L. Van Trees Detection, Estimation and Modulation Theory. New York: Wiley 1968

[8] H. Wymeersch Iterative Receiver Design. Cambridge: University Press, 2007

[9] L. Bahl, J. Cocke, F. Jelinek, and J. Raviv, "Optimal Decoding of Linear Codes for Minimizing Symbol Error Rate," IEEE Trans. Inform. Theory, vol. 20 , no. 2 , pp. 284-287, Mar. 1974

[10] A. D. Andrea, U. Mengali and R. Reggiannini , "The Modified CramerRao Bound and its Applications to Synchronization Problems," IEEE Trans. Commun., vol. 42, no. 234, pp. 1391-1399, Feb.-Apr. 1994

[11] S. Lin and D. Costello, Error Control Coding, 2nd ed. Pearson Education Inc., 2004

[12] M. Moeneclaey, "On the True and the Modified Cramer-Rao Bounds for the Estimation of a Scalar Parameter in the Presence of Nuisance Parameters," IEEE Trans. Commun., vol. 46, no. 11, pp. 1536-1544, Nov. 1998 